\title{
Linx
}

Revue des linguistes de l'université Paris X Nanterre

$50 \mid 2004$

Variation sémantique et syntaxique des unités lexicales : étude de six verbes français

\section{Prémices d'une description du sens du verbe tourner (dans ses emplois intransitifs)}

Danielle Leeman

\section{CpenEdition}

Journals

Édition électronique

URL : http://journals.openedition.org/linx/136

DOI : $10.4000 / \operatorname{linx} .136$

ISSN : 2118-9692

Éditeur

Presses universitaires de Paris Nanterre

Édition imprimée

Date de publication : 1 juin 2004

Pagination : 33-52

ISSN : 0246-8743

Référence électronique

Danielle Leeman, « Prémices d'une description du sens du verbe tourner (dans ses emplois intransitifs) », Linx [En ligne], 50 | 2004, mis en ligne le 27 janvier 2011, consulté le 19 avril 2019. URL http://journals.openedition.org/linx/136; DOI : 10.4000/linx.136 


\title{
Prémices d'une description du sens du verbe tourner (dans ses emplois intransitifs)
}

\author{
Danielle Leeman \\ Université Paris $X$ - Nanterre \\ UMR 7114 (MoDyCo)
}

\begin{abstract}
Que vous mouriez demain n'a aucune importance. Moi de même. On le sait, la vie est une fête qui tourne mal. Doit-on pour autant s'arrêter de danser? (Henri Alloy, Mère est formidable, La Tour d'Oysel, 2003,
\end{abstract} p. 62)

\begin{abstract}
Dans ce titre, le terme «prémices » n'est pas de fausse modestie : il signale d'emblée explicitement que ce qui suit n'est que le témoignage provisoire d'une initiation, d'un apprentissage, que m'ont permis, en m'accueillant dans leur groupe de recherche, les autres contributeurs de ce numéro ainsi que Denis Paillard et Pierre Péroz. Je les remercie pour leur amitié, leur patience dans leurs réponses à mes questions de débutante, leur ouverture d'esprit et le respect qu'ils ont pour le travail d'autrui, que révèle la place qu'ils m'ont réservée dans ce numéro de Linx.

Je commencerai par rappeler les grandes lignes du cadre théorique dans lequel l'étude a été menée, pour établir les similitudes et les différences entre les principes généraux qui guident habituellernement mon travail et ceux des chercheurs s'inscrivant dans la Théorie des Opérations Prédicatives et Énonciatives ( TOPE ») d'Antoine Culioli ${ }^{1}$. Puis je centrerai le propos sur la démarche de description du verbe tourner, considéré essentiellement dans ses emplois intransitifs tels qu'exemplifiés par

\footnotetext{
${ }^{1}$ Il ne s'agit pas d'une leçon de sémantique non démontrée, ainsi que me le reproche amènement l'un des relecteurs anonymes, mais de l'explicitation des principes généraux qui guident la description dont je rappelle, par souci d'honnêteté, en quoi ils ne se conforment pas au modèle «TOPE »-je ne vois pas qu'on puisse en exiger la démonstration préalable.
} 
les outils lexicographiques, mon objectif étant d'aboutir à une définition sémantique générale subsumant les différentes acceptions observées en discours.

\section{Principes théoriques généraux}

L'hypothèse de base est que le mot en langue est l'association d'un signifié et d'un signifiant (F. de Saussure), complétée de la conception guillaumienne suivant laquelle le mot est défini par un ensemble de propriétés formelles (i.e. morphologiques et syntaxiques) et sémantiques qui contiennent en germe et annoncent les configurations (formelles et sémantiques) dans lesquelles il est susceptible d'entrer autrement dit, la structure de la phrase est virtuellement contenue dans l'identité des mots qui la composent. Le point de vue culiolien confirme ces principes généraux et leur donne un cadre théorique et méthodologique précis; il suppose deux thèses corrélées: d'une part «le lexique intègre ses constructions» et d'autre part "non seulement le co-texte mais aussi la variation sont intégrés dans l'identité lexicale » ( $\mathrm{S}$. de Vogüé et D. Paillard, 1997 : 43), d'où le programme de description : réduire «à une forme schématique unique » «l'ensemble des co-textualisations possibles d'une unité donnée» (op. cit. : 44). Si la première thèse est aussi, à la suite de Z. Harris, celle de M. Gross (définissant la théorie dite du «Lexique-grammaire »), les travaux menés dans le cadre du LADL ont pour objectif essentiel de mettre au jour les propriétés syntaxiques et distributionnelles du mot à partir du corpus des phrases élémentaires dans lesquelles il peut entrer, sans en tirer (l'hypothèse d') une identité sémantique qui rendrait compte en retour des effets polysémiques observables dans les divers emplois, et des compatibilités et incompatibilités constructionnelles ou cotextuelles ${ }^{2}$. De même, selon la théorie X-barre rendant compte des structures phrastiques dans le cadre générativiste, les unités syntaxiques (syntagmes, phrases...) sont décrites à partir des constructions admises par les «têtes» qui définissent leur «projection maximale »; le sens du verbe, en particulier, est assimilé à la distribution des rôles thématiques qu'il suppose à ses arguments. La construction est un donné, enregistré comme tel, sans que s'opère une réflexion sur un sens qu'elle serait susceptible de véhiculer ni sur le lien qui pourrait exister entre les différents emplois du même verbe. La définition même du statut d'«argument» reste d'ailleurs problématique : le critère de non-suppression est présenté comme nécessaire et suffisant (ainsi rencontrer doit-il être suivi d'un complément: on ne peut dire simplement $*$ Je rencontre), mais il ne permet en fait pas de dire que les robes dans Je regarde les robes est un argument du verbe, la vendeuse dans le magasin pouvant dire à la visiteuse simplement Je vous laisse regarder, ou la cliente répondre seulement Je regarde à une question telle que Je peux vous aider? La nécessité sémantique ( «si on rencontre, c’est nécessairement quelqu'un ou quelque chose »: rencontrer «appelle» un complément) n'est pas corrélable à une nécessité syntaxique (on a aussi l'implication «si on regarde, c'est nécessairement quelqu'un ou

\footnotetext{
${ }^{2}$ Cf. à l'inverse : «c'est parce que suivre est ce qu'il est, a la valeur qu'il a, qu'il ne peut pas se construire avec $\grave{a}[\ldots]$ Autrement dit, la variation syntaxique doit être explicable à partir des propriétés intrinsèques du verbe considéré, elle est en droit réductible à ces propriétés » (S. de Vogüé et D. Paillard, op. cit. : 48).
} 
quelque chose» mais regarder n'exige pas un complément) $)^{3}$, ce qui est l'une des justifications du principe de l'autonomie de la syntaxe (relativement au sens) - lequel s'oppose à l'hypothèse saussuriano-guillaumienne.

Rappelons que, pour F. de Saussure, il y a dans la langue du radicalement arbitraire (par exemple vingt et un pour parler de «vingt» ou de «un») et du partiellement arbitraire (vingt et un est relativement motivé, une fois admise la correspondance (arbitraire) entre vingt et « vingt» ou un et « un »). L'arbitraire absolu est dans le lexique simple, la motivation est dans la construction (morphologique ou syntaxique) :

«[...] on pourrait dire que les langues où [l'immotivé] atteint son maximum sont plus lexicologiques, et celles où il s'abaisse au minimum plus grammaticales. Non que « lexique » et « arbitraire » d'une part, « grammaire » et «motivation relative» de l'autre soient toujours synonymes, mais il y a quelque chose de commun dans le principe. Ce sont comme deux pôles entre lesquels se meut tout le système, deux courants opposés qui se partagent le mouvement de la langue: la tendance à employer l'instrument lexicologique, le signe immotivé, et la préférence accordée à l'instrument grammatical, c'est-à-dire à la règle de construction ». (F. de Saussure, $1916: 183)$

G. Guillaume pose également une relation non arbitraire entre structure sémiologique (les formes significatives) et structure psychique (les signifiés) de la langue, telle que leur « congruence » est réciproque. Dans cette perspective, aussi bien la catégorisation syntaxique (D. Leeman, 2001 in 2003) que la construction (D. Leeman, 2000 in 2004) ont un sens, de même que l'ensemble des propriétés distributionnelles et syntaxiques (D. Leeman, 2002): il n'est pas indifférent pour l'identité du verbe (en langue) qu'il soit intransitif, transitif, ou les deux ${ }^{4}$, ni que, transitif, il le soit direct ou indirect ${ }^{5}$, qu'il exige ou non la présence de son complément $^{6}$, permette ou non la formulation passive ${ }^{7}$, etc. - autrement dit, ce n'est pas parce que l'on n'a pas d'idée précise sur le sens de la forme qu'elle n'en a pas un ou qu'elle n'est pas pertinente pour le sens qu'elle véhicule.

Le sens ainsi conçu n'est ni d'ordre référentiel (dans la mise en correspondance entre un mot, vu comme une simple suite matérielle, et un «objet du monde », selon

\footnotetext{
${ }^{3}$ Cf. à ce sujet O. Bonami (1999).

${ }^{4}$ Ainsi, le fait que l'on ait J'ai cassé le vase et Le vase a cassé mais non parallèlement J'ai brisé le vase et * Le vase a brisé, doit être considéré comme significatif dans l'opposition des deux verbes - bien qu'on ne voie pas, a priori, en quoi.

${ }^{5}$ Goûte ma tarte et Goûte à ma tarte peuvent paraître quasi synonymes (cf. J.-P. Boons et al., 1976: 66), ce que dément l'appréhension, même purement intuitive, de Gô̂ter les joies de l'amour comparé à Goûter aux joies de l'amour (on peut sans contradiction dire que l'on a goûté aux joies de l'amour sans goûter les joies de l'amour) - cf. aussi C. Vandeloise (1987 et 1993), sur toucher et toucher à.

${ }^{6}$ Il lit peut signifier «il sait lire» aussi bien que «il est en train de lire» mais Il lit un roman ne peut correspondre à l'énoncé d'une propriété : si cette analyse est généralisable, elle signifie que rencontrer ne se prête pas à la formulation d'une propriété - ce qui fait partie de son identité sémantique.

${ }^{7}$ Cf. Langages, 109 : Sur le passif, dirigé par G. Gross (1993).
} 
le terme de J.-C. Milner), ni d'ordre conceptuel (le segment purement formel que constitue le mot étant cette fois associé à la chose telle qu'on se la représente mentalement) : il est institué par la langue elle-même, dans l'interrelation des formes qui organise son système - «le sens des mots et des textes n'est pas extérieur à la langue mais relève d'un ordre propre qui n'est le décalquage ni d'une pensée, ni d'un référent externe»(J.-J. Franckel, 2003) ${ }^{8}$. Certes le sens est de quelque manière «branché » sur le réel objectif, conceptuel, culturel (P. Cadiot \& F. Nemo, 1997, G. Kleiber, 1997, F. Lebas \& P. Cadiot, 2003) ainsi qu'en témoigne le fait que la langue nous permet de parler du monde et de progresser dans sa connaissance, mais l'établissement de ces relations relève d'une autre tâche que celle du grammairien (ainsi Z. Harris (1988) distingue-t-il entre "meaning»- le rapport de la langue avec le monde - et « information » - ce qu'institue le système des formes linguistiques ellesmêmes).

Ce postulat me paraît entraîner une certaine démarche méthodologique, qui est de partir de l'observation des formes (morphologiques et syntaxiques), afin de tâcher d'en tirer des hypothèses pour la définition de leur signifié. Pourtant, la description des verbes déjà traités dans une optique culiolienne ne procède pas ainsi, s'attachant plutôt aux variations distributionnelles : c'est qu'une même construction (par exemple intransitive) n'est jamais biunivoquement associée à un sens, et que c'est surtout le choix lexical qui, dans une structure donnée, fait apparaitre les diverses acceptions (ainsi pour jouer (C. Romero-Lopes, 2002 : 63) : son charme a joué, les enfants ont joué, le bois a joué, ce facteur a joué). La définition de la «forme schématique », qui décrit le sens du mot (en langue), ne s'articule pas directement à sa syntaxe, ni ne permet « de prédire ni de rendre compte des constructions syntaxiques dans lesquelles entre l'unité » (J.-J. Franckel, 2002: 15), au point que certains peuvent conclure « Il existe de ce point de vue une autonomie de la syntaxe » (ibid. $)^{9}$.

Je ne crois pas que l'on ait là une opposition fondamentale et irréductible entre deux démarches (celle qui s'inscrit dans la ligne de Z. Harris à la suite de F. de Saussure et G. Guillaume, et celle qui s'inscrit dans la ligne d'A. Culioli) : j'interprète la mise à l'écart de la syntaxe (et de la morphologie), dans l'élaboration de la forme schématique et la conception de son fonctionnement, comme un principe heuristique provisoire issu du constat que, au point où l'on en est, on ne dispose pas d'une interprétation des constructions susceptible de fournir la base de l'analyse sémantique des verbes. L'approche harrissienne part bien des formes, mais non dotées de signifié - on enregistre une différence ou une similitude, que l'on essaie de transposer sur le plan sémantique, sans toujours pouvoir y parvenir (D. Leeman, 1999); en revanche, l'approche culiolienne procède à des comparaisons de combinaisons distributionnelles qui, en tant que telles, se prêtent mieux à l'exercice de l'intuition : on peut saisir et commenter plus directement ce qui distingue son charme a

\footnotetext{
${ }^{8}$ Cf. aussi J.-J. Franckel (1998). Pour une présentation critique de l'ensemble des sémantiques contemporaines (leurs présupposés, leurs rattachements théoriques et leurs filiations philosophiques plus ou moins conscients), cf. F. Rastier en particulier (1991 rééd. 2001). L. Melis et P. Desmet (2000) font le point sur les diverses approches syntaxiques du vingtième siècle. J.-J. Franckel (2002) procède plus spécialement à une mise au point concernant la théorie culiolienne.

${ }^{9}$ Cette position n'est néanmoins pas également partagée, comme le montre la citation en note 2.
} 
joué et les enfants ont joué, par le biais de synonymes et de gloses, que ce qui rapproche les deux énoncés (qui correspondrait à leur identité structurale). Mais cela ne revient pas à dire que la syntaxe (ou la morphologie) n'a pas de sens ni de rôle sémantique, ainsi qu'en témoigne la démonstration de S. de Vogüé (1991) sur le sens des positions, qui aboutit à la proposition d'une identité sémantique de la fonction de complément d'objet. Pour l'instant, le modèle articule en trois composantes l'identité du verbe : la forme schématique en donne le sens, le schéma de lexis la structure actancielle (argumentale) et le schéma syntaxique la (ou les) construction(s). Il n'y a pas de correspondance terme à terme entre les trois ; ainsi, il arrive la même chose à la clé, que l'on dise Il tourna la clé dans la serrure ou Une clé tourna doucement dans la serrure, mais «l'agent», nécessairement représenté dans le schéma de lexis (en tant que "source» de ce qui arrive à la clé), ne le sera pas forcément dans la forme schématique, si cette dernière se concentre sur ce qui arrive à la clé (cf. sur ces questions, D. Paillard (2000 in 2002) par exemple).

La démarche ici adoptée consistera à voir quels emplois les différences de formes (morphologiques et syntaxiques) séparent, à partir de l'intuition sémantique minimale nécessaire au jugement de paraphrase ou de non-paraphrase - sans lequel aucun travail linguistique n'est possible -, vérifiée autant que possible par un retour aux formes : par exemple, tourner dans la voiture a tourné et le lait a tourné n'a a priori pas la même interprétation, ce que corrobore le fait que, dans le premier cas, on peut dire que la voiture a pris le tournant mais non, dans le second, que * le lait a pris un tournant. Du fait que, comme on vient de le reconnaitre, on ne dispose pas d'hypothèse concernant l'identité des constructions morphologiques ou syntaxiques, on commentera, dans un second temps, chaque emploi d'un point de vue uniquement notionnel ${ }^{10}$, avec l'objectif de fournir de tourner une définition unitaire (au nom du principe de naturalité posant (en langue) une relation biunivoque entre signifiant et signifié). Il s'agit d'essayer de voir ce qui est commun aux énoncés et constitue l'identité propre de tourner, de façon à repérer quelle interprétation à la fois il exige de et affecte à son cotexte - cette identité étant vue comme le rôle que joue le mot dans la construction du sens des énoncés où il apparait.

Contrairement aux dictionnaires (Grand Larousse de la langue française - désormais GLLF - et Trésor de la langue française - dorénavant TLF - par exemple), je commencerai par l'emploi intransitif (et m'y bornerai par manque de place), du fait que certains des emplois transitifs sont à analyser comme des factitifs; ainsi le TLF définit-il «I. Emploi transitif» d'abord par «faire mouvoir quelque chose autour d'un axe ", l'un des exemples illustratifs étant Tourner la clé dans la serrure, à comparer avec La clé tourne dans la serrure, qui exemplifie l'emploi intransitif. Cela ne veut pas dire que

\footnotetext{
${ }^{10}$ L'analyse notionnelle ne me parait pas devoir être ramenée à la "constitution référentielle » (F. Lebas \& P. Cadiot, op. cit. : 15), dans la mesure où il s'agit de confronter des usages de mots à d'autres usages de mots, et non de faire correspondre un mot en emploi avec un objet ou une situation, un peu comme lorsque, ayant à traduire voir en anglais, on se demande si c'est look ou see qui sera le plus approprié : pour ce faire, on ne cherche pas quels types de perception dans la réalité désignent voir, look ou see, mais plutôt quels types de formulation se correspondent le plus fidèlement a priori dans les deux systèmes linguistiques étant donné le cotexte dans lequel s'inscrit l'item à traduire - du moins est-ce ainsi que j’utilise les dictionnaires !
} 
tous les emplois transitifs de tourner soient des factitifs, ni que je considère les deux constructions synonymes : c'est un simple choix heuristique minimalement justifié, toutefois étayé par la conclusion de J. Dubois et F. Dubois-Charlier (2003: 3) issue de leur description syntaxico-sémantique de plus de 12.000 verbes (J. Dubois et F. Dubois-Charlier, 1996): "Les structures syntaxiques verbales de base à partir desquelles se développent en français les autres structures sont des phrases à $\mathrm{V}$ à un seul argument, dites intransitives ».

\section{Inventaire des emplois de tourner (intransitif)}

On part des exemples tels qu'énumérés par le GLLF mais sans nécessairement suivre sa progression ni opérer les mêmes rassemblements, du fait que l'on cherche à les regrouper sur des bases formelles (par le biais des similitudes de construction ou de paraphrases à l'aide de noms ou d'adjectifs apparentés morphologiquement) ${ }^{11}$; est adoptée une représentation «surfaciste» des énoncés, où $\mathrm{N}_{0}$ correspond au sujet syntaxique et $\mathrm{N}_{1}$, voire $\mathrm{N}_{2}, \mathrm{au}(\mathrm{x})$ complément(s) éventuel(s) - à la manière, donc, des conventions utilisées par M. Gross (1975) par exemple. On parlera ${ }^{12}$ de "procès dynamique » lorsque le verbe autorise la périphrase être en train de et la reprise en faire, et de "procès statique » dans le cas contraire; le procès sera dit «imperfectif» ou «atélique » si le verbe est modifiable par un circonstant tel que éternellement, durant des lustres, «perfectif» ou « télique » si le modifieur peut être de type en une heure, en quelques secondes, « ponctuel» lorsque le verbe ne supporte ni l'un ni l'autre de ces compléments mais admet par exemple à midi, à ce moment précis; le trait [changement] n'a de définition que sémantique, à ma connaissance : il s'agit de déterminer si $\mathrm{N}_{0}$ est dans une certaine situation ou un certain état, avant le procès, différents de ceux dans lesquels il se trouve une fois le procès accompli (J. François, 1989). Avec tourner toutefois, l'état résultatif différent de l'état initial est susceptible de se formuler de façon particulière : par le participe passé adjectivé (La sauce a tourné : elle est tournée / une sauce tournée).

\subsection{No tourne autour de $N_{1}$}

Dans cette construction, on considère que autour de $N_{1}$ est un complément (obligatoire), l'énoncé La terre tourne ne se comprenant pas comme La terre tourne autour du soleil, ni Le boulevard tourne comme Le boulevard tourne autour de la ville.

- Emplois dynamiques

Le verbe est paraphrasable par faire un ou des tour(s), ou tourne autour de $N_{1}$ par fait le tour de $\mathrm{N}_{1}$; le procès est imperfectif ou perfectif et l'on n'a pas le trait [changement] :

\footnotetext{
${ }^{11}$ Je m'écarte, ce faisant, de la démarche adoptée dans la plupart des autres articles de ce numéro, de deux manières : d'une part, le corpus est uniquement lexicographique, et simplifié par rapport à ce que l'on trouverait dans Frantext; d'autre part, l'investigation préalable est d'ordre morphosyntaxique.

${ }^{12}$ Les termes que j'emploie (cf. par exemple J. François, 1989) ne sont pas ceux de la théorie culiolienne : je m'en tiens à la tradition faute de réellement maitriser pour le moment la tripartition « discret-dense-compact» (A. Culioli, 1982a, D. Paillard, 1988).
} 
(1) a. La terre tourne autour du soleil, fait des tours autour du soleil, fait le tour du soleil.

b. La terre tourne autour du soleil éternellement.

c. La terre tourne autour du soleil en une année.

d. Les ombres tournaient autour des mégalithes. (elle ne se trouvent pas dans un état différent avant et après avoir tourné)

L'interprétation télique (en une année) suppose un nombre déterminé de tours tels que le terme auquel $\mathrm{N}_{0}$ aboutit coïncide avec son point de départ ; l'interprétation atélique (éternellement) suppose un recommencement du même parcours : le(s) tour(s) dessine(nt) une configuration fermée, autonome en ce qu'elle se définit elle-même. $\mathrm{N}_{0}$ est l'objet d'une variation qui ne fait sens que pour un observateur extérieur (par exemple l'astronome qui interprète le mouvement de la terre en le référant au soleil).

Dans le cas de (1.e), le procès ne peut être qu'imperfectif et les paraphrases faire des tours, faire le tour de apparaissent inappropriées :

(1) e. Tout tourne autour de moi. Je vois tout tourner!

Le complément autour de $N_{1}$ n'est plus obligatoire; le constat est connoté négativement : il a valeur détrimentale ("je ne me sens pas bien », « je ne suis pas dans mon état normal») : l'énonciateur juge que ce qui tourne - ou du moins lui apparaît tourner - ne devrait pas tourner. Comme dans les exemples (1.a-d), l'activité de $\mathrm{N}_{0}$ est définie par rapport à un repère $\mathrm{N}_{1}$ (le soleil, les mégalithes, mol) mais ce centre n'en constitue pas la finalité : $\mathrm{N}_{0}$ tourne autour de $\mathrm{N}_{1}$ sans chercher à le rejoindre ou à s'en éloigner, par exemple.

On retrouve ces valeurs dans les emplois (1.f-g), également imperfectifs ; mais ils se prêtent mal aux paraphrases faire des tours, faire le tour de et ne supposent pas nécessairement une configuration fermée :

(1) f. Tourner autour de quelqu'un (" ne pas oser l'aborder directement»), Tourner autour d'une femme (« s'intéresser à elle, lui faire la cour »)

g. Tourner autour du pot, autour de la question

Le complément dénote ce qui fonde que $\mathrm{N}_{0}$ tourne, mais cette activité reste atélique relativement à ce $\mathrm{N}_{1}: \mathrm{N}_{0}$ n'atteint pas $\mathrm{N}_{1}$ - d'où, là encore, un possible effet détrimental.

- Emploi statiques

Dans le cas de (1.h-j), le procès est statique et la paraphrase faire le tour de seule admissible - également en une interprétation statique :

(1) h. L'escalier tourne autour de la cage d'ascenseur.

i. Un boulevard tourne autour de la ville.

j. Un bracelet d'or en spirale tourne autour du bras bronzé de notre hôtesse.

Est donnée une propriété de $\mathrm{N}_{0}$, mais toujours décrivant le fait que $\mathrm{N}_{0}$ obéit à une configuration déterminée $\left(\mathrm{N}_{0}\right.$ est censé suivre une certaine ligne, imposée par la 
forme de $\mathrm{N}_{1}$ ), qui n'est pas, ici non plus, nécessairement fermée (ainsi les deux bouts du bracelet en spirale ne se rejoignent pas).

Dans (1.k), la paraphrase faire le tour de est elle-même inacceptable, mais le sens est aussi celui d'un $\mathrm{N}_{1}$ vu comme ce qui à la fois engendre l'activité caractéristique de $\mathrm{N}_{0}$ et en constitue la visée, sans que le processus aboutisse à l'atteinte du but (les religions sont présentées comme discutant indéfiniment du problème sans le résoudre) :

\section{(1) k. Chaque religion tourne autour des notions d'innocence et de culpabilité. (Camus)}

En résumé, dans $N_{0}$ tourne autour de $N_{1}$, tourner définit une certaine activité (emploi dynamique) ou une certaine identité (emploi statique) de $\mathrm{N}_{0}$ en relation avec $\mathrm{N}_{1}: \mathrm{N}_{1}$ constitue une sorte de centre de gravité qui donne son sens à la variation de $\mathrm{N}_{0}$ dans son déplacement (la terre, les ombres), dans sa forme (l'escalier, le boulevard), dans son contenu (la religion), mais sans que cette relation soit jamais une coïncidence ni sanctionnée par un résultat (le procès est imperfectif) - sinon celui d'un retour au point de départ, lorsque le procès est télique; tourner décrit donc une manifestation (statique ou dynamique) qui constitue sa propre finalité - dans les emplois (1.f, g, k), le fait que la source (ou cause) du «tourner» puisse être aussi sa visée confirme le caractère circulaire de cette manifestation.

\subsection{No tourne (sur soi-même)}

L'exemple emblématique est ici (2.a), paraphrasable par Elle fait des tours / un tour sur elle-même :

\section{(2) a. La terre tourne (sur elle-même).}

Le procès est dynamique (à moins que, comme en (2.a), on n'énonce une propriété de $\mathrm{N}_{0}$ ) mais il apparait pouvoir être aussi bien imperfectif, perfectif ou ponctuel :

(2) b. Les manèges tournent des heures durant.

c. La terre tourne sur elle-même en vingt-quatre heures.

d. Une clé a tourné dans la serrure (?? en une seconde vs à cet instant précis).

Le $\mathrm{N}_{1}$ correspond implicitement (par métonymie) ou explicitement à une partie constitutive du référent de $\mathrm{N}_{0}$ (la préposition sur introduit l'axe, le pivot qui permet à $\mathrm{N}_{0}$ de tourner) :

\section{(2) e. Tourner sur ses talons}

L'interprétation est semblable à celle de l'emploi précédent: un parcours complet avec retour (au moins tendanciel) au point de départ.

Notons que dans cet emploi du verbe, sur - quoique d'ordre spatial - ne peut guère s'analyser dans les termes de C. Vandeloise (1986) ou P. Dendale et W. De Mulder (1997, 1998). S'il y a en effet "position sur l'axe vertical», ce n'est certainement pas de telle sorte que la terre (la «cible») serait «plus haute » qu'ellemême (le "site»), et puisque les deux se confondent, on ne peut pas non plus en l'occurrence dire que la cible soit «plus petite» que le site, qu'il y ait «contact» ou 
«adhérence» entre la cible et le site, ni que le site «s'oppose à l'action de la pesanteur» sur la cible. Certes le site ne paraît pas non plus être «conceptualisé comme contenant» (trait ajouté par P. Dendale \& W. De Mulder (1997 : 218) pour distinguer sur de dans), mais si ce sème permet de justifier pourquoi on n'a pas *La terre tourne dans elle-même, il ne peut servir à expliquer pourquoi on n'a pas non plus par exemple * La terre tourne contre elle-même. On pourrait admettre en revanche, à l'aide de D. Paillard (2000 in 2002 : 58), que sur en (2') instaure la terre elle-même comme le lieu principal de l'accomplissement du procès tourner par la terre (elle-même en est à la fois le lieu et la source). On opposera de même (2') et (2") :

(2') La toupie tourne sur elle-même.

(sur institue elle-même comme le lieu inhérent au tournement)

(2") La toupie tourne sur le guéridon.

(sur spécifie le milieu dans lequel se déroule le tournement)

En (2'), la préposition établit la relation, tandis qu'en (2') il existe une relation entre la toupie et le guéridon indépendante de sur, ce qui se voit au fait qu'elle peut être précisée par d'autres prépositions (sous, près de, contre...).

Dans (2.f), le causatif paraît nécessaire (Les tables tournent parait a priori étrange, sauf à retrouver l'interprétation [1.e]) :

(2) f. Faire tourner les tables

On peut en conclure que tourner suppose du référent de $\mathrm{N}_{0}$ qu'il possède en soi la propriété de se mouvoir de manière autonome (une clé tourna dans la serrure en (2.d) s'interprète de même comme "quelqu'un fit tourner la clé dans la serrure »).

La construction que l'on va maintenant étudier recouvre deux cas de figure, selon que $\mathrm{N}_{1}$ s'analyse comme un ajout (un «complément scénique», dans la terminologie de J.-P. Boons, A. Guillet, C. Leclère, 1976) ou comme un complément de verbe : Le bus tourne sur la place peut en effet signifier respectivement, soit « Le bus est sur la place et il tourne » (la place est le lieu où il se produit que le bus tourne), soit «Le bus n'est pas sur la place mais il s'y engage » (la place est le point d'aboutissement du tournant opéré par le bus).

\subsection{No tourne quelque part (complément "scénique»)}

La préposition introduisant $\mathrm{N}_{1}$ est en l'occurrence variable (contrairement à autour de ou sur en 2.1. et 2.2.) et ne permet pas toujours l'équivalence avec faire le tour de (L'avion fait le tour de la ville ne constitue pas une paraphrase de L'avion tourne au-dessus de la ville):

(3) a. Il n'a pas cessé de tourner dans sa chambre.

b. Il a tourné dix minutes derrière le pâté de maison avant de pouvoir garer sa voiture.

c. L'avion tourne au-dessus de la ville en attendant de pouvoir se poser.

Le procès est dynamique, imperfectif, et n'implique pas de [changement]; l'interprétation atélique, là encore, suppose le recommencement d'un même parcours 
revenant à son point de départ : $\mathrm{N}_{1}$ définit ce qui impose l'itinéraire, mais c'est tourner qui implique la «circularité » (au sens où l'on parle d'un raisonnement circulaire) ${ }^{13}$, comme en témoigne l'emploi des mêmes prépositions que ci-dessus avec d'autres verbes :

\footnotetext{
(3) a'. Il n'a pas cessé de marcher dans sa chambre.

b'. Il a roulé dix minutes derrière le pâté de maisons.

c'. Un avion passe dans le ciel au-dessus de nos têtes.
}

Par opposition à (3.a), (3.a') n'implique pas de configuration particulière du déplacement (si l'on peut marcher de long en large, on ne peut tourner de long en large). De même, en comparaison avec (3.b), (3.b') ne contient pas l'idée d'un retour à un point de départ (il aurait aussi pu rouler tout droit pendant dix minutes, mais on ne saurait tourner tout droit). Pareillement, l'avion qui passe ou qui vole au-dessus de la ville ne fait qu'en traverser l'espace aérien (il peut passer ou voler d'est en ouest, précision non applicable à tourner).

Le cas (3.d) illustre aussi le cas d'un complément « scénique » mais se distingue des précédents en ce que le verbe est associable au nom tournée :

\section{(3) d. Un agent commercial qui tourne dans la région de Marseille}

Il s'agit cependant là encore d'un parcours préétabli (tels lieux à visiter, telles personnes à rencontrer) qui, par comparaison avec circuler (L'agent circule dans la région de Marseille), me paraît supposer un retour au point de départ: l'agent qui circule, va ou circule d'un point à un autre, on ne pourrait en dire qu'il tourne d'un point à un autre. Tous ces verbes, dont la substitution à tourner n'engage pas, dans le cotexte considéré, une modification sémantique radicale a priori, s'opposent à lui par le fait qu'on peut leur associer un terme que permet d'atteindre le déplacement: on peut marcher, rouler, circuler jusqu'à un certain lieu ou d'un endroit à un autre, mais tourner refuse ce type de distribution - sauf à imaginer que l'entité concernée opère le parcours en faisant des tours (au sens 2.1. ou 2.2.) : Il a tourné jusqu'à sa chambre, «il y est allé en tournant». Ainsi se confirme-t-il que tourner suppose une configuration autonome en ce qu'elle contient en elle-même - et non dans un repère extérieur - sa propre fin.

\subsection{No tourne quelque part (complément de verbe)}

Ce qui appuie l'idée (cf. note 13) que ce n'est pas la circularité (au sens d'un "parcours en forme de cercle») qui caractérise l'identité de tourner, ni la fermeture du parcours, c'est que Il tourne peut s'interpréter comme «il prend un/le tournant» s'il s'agit d'un procès dynamique ou comme « il fait un tournant» dans le cas d'un chemin

\footnotetext{
${ }^{13}$ Le verbe ne suppose pas nécessairement un parcours ayant la forme de la figure géométrique : tourner en rond n'apparait pas tautologique et à l'inverse les mouches qui tournent devant, autour ou au-dessus d'un plat de viande peuvent chacune avoir leur propre itinéraire, successivement se rapprocher ou s'éloigner de la cible, s'élever dans les airs ou plonger vers la table, aller de gauche à droite, etc. ; le parcours n'est donc pas circulaire (en ceci qu'il décrirait un cercle) mais déterminé par le plat de viande auquel cependant il n'aboutit pas: la finalité extérieure du procès reste hors d'atteinte mais c'est elle qui lui donne son sens.
} 
(procès statique), qui n’évoquent pas un «cercle». Dans un énoncé comme (4.a), la préposition introduit un complément de verbe (ils passent d'un endroit qui n'est pas une allée à un endroit qui est une allée, et de même moi se retrouve dans les taillis alors qu'il n'y était pas avant de tourner) :

(4) a. Ils tournent dans une allée et moi dans le taillis. (Marivaux)

Le procès est dynamique et présente le trait [changement], il est perfectif ou ponctuel : il n'y a pas ici une activité continue mais au contraire une rupture, une discontinuité ; il n'y a pas non plus de retour - même tendanciel - au point de départ. Il en va de même pour:

(4) b. Au prochain carrefour, vous tournerez à gauche.

c. Il sourit tout à coup : la fortune tournait de son côté et ses yeux brillèrent. (Musset)

Ce qui relie toutefois cet emploi aux trois autres, c'est qu'il s'agit pour $\mathrm{N}_{0}$ d'adopter une certaine configuration définie par $\mathrm{N}_{1}$ : le chemin impose à celui qui l'emprunte un certain parcours. Si l'on compare en effet à tourner les verbes perfectifs ou ponctuels (associés à un complément locatif) qu'on pourrait lui substituer dans ces cotextes : aboutir, aller, arriver, entrer, courir, passer..., la différence est que ces derniers ne contiennent en eux-mêmes que le changement de direction de $\mathrm{N}_{0}$, tandis que tourner inclut de surcroît la modification de la forme du chemin lui-même. Si l'on tourne dans une allée (4.a), c'est que la voie que l'on emprunte comporte un tournant qui débouche sur cette allée, alors que Ils vont / aboutissent / arrivent dans une allée ne suppose pas une configuration particulière du chemin suivi jusqu'ici - une forme propre qui inclurait intrinsèquement le changement de direction.

De même, courir ou passer dans le taillis implique l'initiative de la part de $\mathrm{N}_{0}$ de transformer son itinéraire, mais tourner dans le taillis implique de plus une rupture prévue par l'itinéraire lui-même - qui contraint donc le déplacement de $\mathrm{N}_{0}$ s'il veut aboutir au taillis. Une formulation comme La fortune vient de son côté entérine la direction que prend la fortune, mais La fortune tourne de son côté (4.c) y ajoute le fait que la fortune elle-même connaît une mutation (pour se diriger de son côté, il lui faut tourner). Le commentaire serait similaire pour Vous prendrez à gauche comparé à Vous tournerez à gauche (4.b), qui ajoute à l'initiative de $\mathrm{N}_{0}$ de prendre à gauche la condition que pour ce faire il doit opérer un tournant. Pareillement, passer ailleurs c'est choisir d'emprunter un chemin autre que celui qui était indiqué, tandis que tourner ailleurs suppose acquis qu'il faut tourner: on se soumet à la bifurcation imposée par le chemin, ailleurs disant que l'on se plie à cette exigence mais en la différant (j'ai manqué l'endroit où il fallait tourner: je tournerai plus loin) - tourner implique que le changement est dans la définition de l'identité du chemin (il lui est interne) et s'impose (de l'extérieur) à l'entité qui se déplace; de fait, la modification peut faire l'objet de l'énoncé d'une propriété :

(4) d. A deux cents mètres, l'avenue tourne.

e. Sachant jusqu'où [les chemins] allaient filer droit, où ils tourneraient. (Proust)

f. Sous les arbres dépenaillés tournent des allées sans mystère. (Colette) 
Si l'avenue, le chemin, l'allée tournent, alors, les empruntant, on doit tourner aussi.

Un autre point commun avec les emplois précédents est celui d'une instabilité, qui n'est pas, comme en (1.e), dans le constat que quelque chose tourne alors que cela ne devrait pas être le cas, mais dans celui que ce qui tourne ne va pas tout droit (contraste qu'exhibe explicitement (4.e)); le tournant suppose un écart dans une trajectoire envisagée ou attendue à partir du chemin parcouru (4.g), ce caractère imprévisible pouvant cacher un danger potentiel (4.h) : $\mathrm{N}_{0}$ peut d'ailleurs référer dans ce cas à des entités capricieuses, versatiles et imprévisibles : la fortune, la chance, le destin, le vent...

(4) g. Être à un tournant de sa carrière. Un tournant de l'bistoire. Une décision qui marque un tournant

(4) h. Attendre quelqu'un à un tournant. Avoir quelqu'un au tournant

Celui qui se trouve concerné par ce qui tourne est dans une situation instable dans la mesure où il n'a pas de prise sur elle : se confirment ainsi les conclusions précédentes selon lesquelles tourner suppose une variation autonome, sans finalité ou point d'aboutissement contrôlable.

\subsection{No tourne à $N_{1} / N_{0}$ tourne en $N_{1}$}

Dans l'emploi 2.4., le complément quelque part peut prendre la forme à gauche, à droite, à l'est (Le vent tourne à l'est), où d'autres prépositions que à sont susceptibles d'apparaittre : vers la gauche, sur la droite. Ici, à est figé et introduit des noms prédicatifs (événement, état, propriété) :

(5) a. Avec l'áge, il tourne à la misanthropie, au mysticisme.

b. Un incident banal qui tourne au tragique

c. La fuite de la droite ennemie tournait à la débâcle. (Malraux)

d. Hier soir, le ciel était d'un bleu qui tournait au noir. (Green)

Une paraphrase par prendre un tour aussi bien que prendre un tournant est possible si le $\mathrm{N}_{1}$ a un correspondant adjectival susceptible de modifier tour et tournant:

(5) a'. Avec l'âge, son caractère adopte un tour mystique / prend un tournant mystique. b'. Un incident banal qui prend un tour tragique / connaît un tournant tragique.

Le procès est perfectif ou ponctuel et débouche sur un changement (l'incident était banal, il devient tragique) :

(5) c'. La fuite de la droite ennemie tourne à la débâcle en quelques instants / à ce moment précis.

Comme en 2.4., tourner décrit une évolution interne, c'est-à-dire non liée à une cause extérieure mais en quelque sorte déjà « programmée » par la nature de $\mathrm{N}_{0}$ : il est dans la nature du lait d'avoir une certaine évolution et de devenir aigre (Le lait tourne à l'aigre). De même, un bleu qui tourne au noir suppose une continuité entre le bleu et le noir, tandis que un bleu qui passe au noir présente la transformation comme un saut à un état différent : on passe à autre chose - de la même manière, en 2.4., passer ailleurs était 
compris comme «prendre un autre chemin que celui que l'on doit suivre». Et dire qu'une idée tourne à l'obsession, c'est signifier qu'une idée déjà prégnante dans l'esprit de la personne devient progressivement son unique préoccupation.

Dans tous les cas, le procès (statique) est un jugement porté sur $\mathrm{N}_{0}$ dont l'observateur interprète la variation : on retrouve l'idée déjà avancée à propos de $\mathrm{La}$ terre tourne autours du soleil en 2.1., selon laquelle c'est l'astronome qui donne un sens au processus dans lequel s'inscrit la terre.

Comme en 2.4. également, cette modification interne que décrit (5) est relativement imprévisible pour l'observateur qui en prend conscience et la qualifie, d'où une possible valeur détrimentale ${ }^{14}$, ainsi que le montrent les exemples et les expressions lexicalisées enregistrés par les dictionnaires :

(5) e. Votre frère aîné allait tourner à l'bébétude et entrer dans les ordres. (Montherlant) f. Tous leurs efforts ont tourné à leur confusion. (Rousseau)

g. Tourner à l'aigre. Tourner à la graisse, à l'buile (prendre une apparence huileuse sous l'effet de la maladie appelée "graisse»). Tourner en eau de boudin. Tourner en bourrique $\mathrm{N}_{1}$ est en:

Lorsque $\mathrm{N}_{1}$ se prête à une identification de $\mathrm{N}_{0}$ à $\mathrm{N}_{1}$, la préposition introduisant

(5) h. L'amour que j'ai pour toi tourne en baine pour elle. (Corneille)

L'amour devient de la haine au terme du procès tourner - qui est donc télique et a le trait [changement] -, la proximité des deux sentiments étant un poncif bien connu.

\subsection{No tourne (d'une certaine manière)}

Dans l'emploi 2.5., le complément à $N_{1}$ ou en $N_{1}$ peut entrer en équivalence avec un adverbe de manière : bien, mal, comme sa ${ }^{15}$ : une discussion qui tourne au vilain ou an vinaigre peut être dite, de manière grossièrement paraphrastique, tourner mal ou de même la fuite de la droite ennemie (5.c) tourner de façon favorable ou défavorable, selon le point de vue. Il y a donc un lien avec les emplois (6) ci-dessous, où cependant le complément est facultatif :

(6) a. L'heure tourne. L'heure a vite tourné.

b. Le moteur tourne à mille tours minute, à plein régime.

c. Les entreprises continuent à tourner.

d. Un service qui tourne rond

e. C'est une affaire qui tourne.

\footnotetext{
${ }^{14}$ La valeur négative n'est cependant pas systématique : le temps peut tourner an beau aussi bien que à la pluie et même dans ce dernier cas, le changement est susceptible d'être interprété positivement, selon le contexte.

15 «Elle aime ce manant, et elle nous menace de tourner comme sa sœur» (Sand, cité par le TLF) : la crainte est qu'elle ne tourne mal.
} 
Ici, on ne peut associer à tourner des formulations avec tour ou tournant et le procès n'est plus perfectif ou ponctuel : on retrouve donc les interprétations atéliques des emplois 2.1. à 2.3. telles que $\mathrm{N}_{0}$ est vu pris dans une activité continue.

Dans le cas du moteur, de l'usine ou des entreprises ${ }^{16}$, tourner sans modifieur met l'accent sur le fonctionnement approprié, harmonieux, de l'ensemble. Ce qui oppose tourner à marcher, dans ces situations, c'est qu'avec tourner est reconnu le fait que chaque partie joue convenablement son rôle en interaction avec les autres - donc à l'intérieur du tout considéré en lui-même -, tandis que marcher porte un jugement global sur le tout, dans sa conformité à une attente (Alors, ça a marché ?) sans prendre en considération les parties; en somme tourner qualifie le fonctionnement, tandis que marcher prend acte de son résultat : une entreprise ou une machine peuvent tourner sans pour autant (bien) marcher, et un moteur tourner à vide - pour rien ; marcher présuppose donc tourner et non l'inverse, comme le montre le test de leur coordination en mais (* Ca tourne mais ça marche vs Ça tourne mais ça ne marche pas) ${ }^{17}$ et enregistre un résultat effectif (* Ca marche à vide). Cependant, cette activité du $\mathrm{N}_{0}$ qui « tourne » en harmonie avec sa fonction peut donner lieu à jugements mitigés : c'est que, si les choses vont comme elles doivent aller, ce bon fonctionnement est purement autotélique (on ne progresse pas, il n'y a pas de finalité extérieure) ; on retrouve cette idée que tourner décrit une activité atéléonomique et donc fermée sur elle-même, qui ne mène nulle part :

(6) f. Ce qui est assez, grave dans une partie des recherches en grammaire générative et transformationnelle, c'est que c'est conduit de telle manière que cela peut «tourner»; or le problème n'est pas que cela tourne ou non, le problème est d'avoir une procédure qui permette d'etablir des interactions entre des domaines qui très souvent [...] nous apparaissent comme disjoints. (A. Culioli, 1979 in 1999 : 82)

Pourquoi le fait que «cela tourne» n'est-il pas jugé pertinent par l'auteur, pourquoi le fait de construire quelque chose qui «tourne » est-il estimé « assez grave » (ce qui paraît paradoxal) ? C'est, d'après le contexte, que ce qui tourne fonctionne sans qu'on en ait la justification, et sans correspondre à la production de résultats intéressants ; ainsi tourner décrit un fonctionnement harmonieux en soi mais qui n'a pas de raison d'être, aux yeux de celui qui emploie le verbe : celui qui dit ça tourne le dit sur un fond d'insatisfaction (l'observateur reconnaît la variation mais refuse de la valider). Ce commentaire vaut pour (6.g):

(6) g. Seul un travail en équipe soigneusement organisé permettra de construire un modèle qui ne soit ni réducteur ni inconsistant. Il y faut des mathématiciens [...]; des linguistes qui n'aient pas une foi ingénue en l'observation directe ou une allégeance à tout ce qui serait formel et "tournerait rond"; des informaticiens [...]; des psycholinguistes [...]. (A. Culioli, 1974 in 1999 : 64)

\footnotetext{
${ }^{16} \mathrm{En}$ (6.e), affaire peut s'appliquer à une entreprise mais aussi avoir un sens beaucoup plus général : C'est une affaire qui tourne est alors lexicalisé (on ne dirait pas dans la même interprétation L'affaire tourne, C'est une affaire qui ne tourne pas / qui tourne bien).

${ }^{17}$ Sur cette interprétation du « test de mais», cf. J.-C. Anscombre (1995: 60).
} 
Là encore, tourner rond n'est pas employé de manière positive, alors qu'on aurait tendance à penser (spécialement par comparaison avec ce qui ne tourne pas rond) que tourner rond est conforme à ce que l'on attend du fonctionnement normal d'un modèle : c'est qu'un modèle peut fort bien remplir son office - ici : rendre compte de ce qu'il formalise - tout en étant « réducteur» ou « inconsistant» - autrement dit en ne servant à rien d'autre qu'à lui-même. Les modèles dont il est question se délimitent un objet tel que peut en rendre compte le formalisme adopté: ils sont donc nécessairement appropriés, mais clos sur eux-mêmes, infalsifiables. (6.h) illustre une restriction similaire (il s'agit de l'Intelligence Artificielle : IA) :

(6) h. Si on la considérait comme une science, on serait inévitablement conduit à perpétuer cette erreur aujourd'bui banale: croire que l'application qui «tourne sous la forme d'un système d'LA valide par là-même les conceptions théoriques qui ont présidé à sa mise en auvre. (F. Rastier, 1991 rééd. 2001 : 36)

\subsection{No tourne, a tourné, est tourné}

Lorsque l'on parle de lait, de beurre, de mayonnaise, de légumes - en somme de denrées périssables -, tourner n'est reliable ni à tour, ni à tournant; il décrit un procès perfectif dont le résultat est formulable par le participe passé adjectivé :

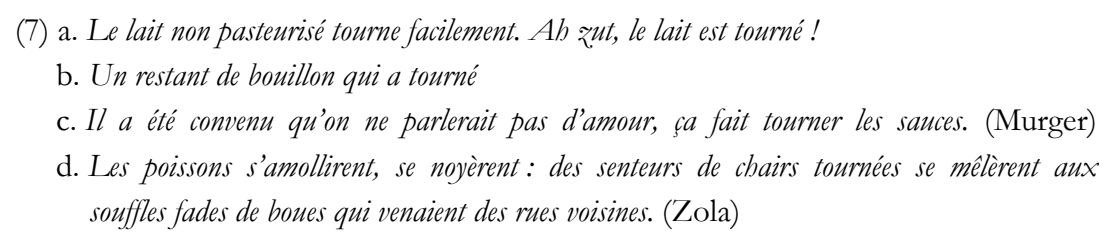

Le GLLF signale que tourner se dit aussi « en parlant du raisin, des fruits : prendre de la couleur en mûrissant» et «en parlant des laitues, des choux : pommer », où le résultat n'apparaît pas détrimental. Comme dans les emplois 2.4. et 2.5., également téliques, le verbe exprime une modification interne, une évolution conforme à une "programmation" naturelle dont le contrôle échappe à un observateur extérieur - qui n'en est pas moins celui qui interprète la variation, lui donne sens en lui attribuant une finalité.

\subsection{Les « restes"}

Si l'intuition permet - éventuellement guidée par la description lexicographique - de ranger les autres emplois sous telle ou telle rubrique sémantique, on ne peut justifier le rapprochement par les critères formels jusqu'ici retenus ; ainsi dans :

(8) a. Son pied a tourné.

le procès est plutôt ponctuel que perfectif (?? Son pied a tourné en une seconde) mais on ne peut guère dire que le pied a fait un tour ou pris un tournant, ni associer au verbe un complément en sur comme en 2.2. (?? Son pied a tourné sur lui-même), ni en quelque part comme en 2.4., ni un adverbe de manière comme en 2.5. ou 2.6., et l'on a aussi 
difficilement ?? Son pied est tourné. Il en va de même pour (8.b), le procès étant cette fois imperfectif :

(8) b. La tête me tourne, je n'y vois plus rien.

ou encore pour :

(8) c. Tu me fais tourner la tête. (E. Piaf)

d. Dès la seconde bouffée, le cour me tourna. (Maupassant)

e. Tourner de l'ail

Tous ces emplois ont en commun de concerner un $\mathrm{N}_{0}$ dit « de partie du corps » et illustrent le cas d'une variation imprévisible et incontrôlable, le plus souvent détrimentale - le cas (8.c) ne paraissant pas correspondre à une situation dommageable...

Dans le cas de Silence, on tourne! ou de Elle tourne beaucoup en ce moment (dit d'une actrice de cinéma), tourner est associable au nom tournage et peut (provisoirement) être considéré comme un emploi absolu du verbe transitif (tourner une scène, tourner beaucoup de films).

\section{Vers une hypothèse de «forme schématique »}

La forme schématique vise à caractériser l'identité du terme dans son apport singulier au sens de l'énoncé et son interaction avec le cotexte (en ceci qu'il donne à interpréter d'une certaine manière les termes qui lui sont co-occurrents). Les commentaires des emplois analysés en 2 . me paraissent déboucher sur la proposition suivante : tourner dit que

Une entité $\mathbf{a}$ est inscrite dans une variation $\mathbf{b}$ définie par la nature propre de $\mathbf{a}$ et en relation avec la nature d'une entité extérieure $\mathbf{c}$ qui peut être un observateur.

Le mot variation est ici préféré à processus, qui peut ne suggérer qu'un procès dynamique (or on a vu que tourner peut aussi correspondre à un procès statique). Dans La terre tourne, il y a une entité a (la terre) prise dans un processus $\mathbf{b}$ (tourner) qui trouve sa source dans la nature de la terre elle-même et ne sort pas de cette logique interne (il n'y a pas de causalité ni de finalité extérieures au procès) - d'où la formulation est inscrite dans. Le commentaire de Le lait tourne serait identique, de même que celui de L'allée tourne - à condition de considérer $\mathbf{b}$ comme statique, restriction que le terme variation me semble permettre d'éviter.

La formulation définie par la nature propre de a correspond au fait que a doit, par ses propriétés constitutives, être susceptible de variation (cf. ce que l'on a dit du nécessaire causatif dans Les tables tournent / Faire tourner les tables), et fait écho au caractère autonome de $\mathbf{b}$ (à l'absence d'une causalité aussi bien que d'une finalité extérieures) : la variation a sa source dans la nature de a et est atéléonomique.

Dans La terre tourne autour du soleil, la terre tourne est décrit par : "une entité a est inscrite dans une variation $\mathbf{b}$ définie par la nature propre de $\mathbf{a} »$ et autour $d u$ soleil spécifie que l'observateur rapporte la variation à l'existence d'une entité $\mathbf{c}(l e$ soleil $:$ le complément indique ce relativement à quoi la terre est vue tourner ; il en va de même 
pour L'escalier tourne autour de la cage d'ascenseur - le procès, ici, étant statique. Dans La clé tourne dans la serrure est spécifié le cadre c (la serrure) de la variation de l'entité a (la clê) ; pour Un bleu qui tourne au noir ou Le bouillon tourne à l'aigre, l'entité c (le noir, l'aigre) correspond à l'interprétation, par l'observateur, de la variation en terme d'une altérité qu'il attribue à $\mathbf{b}$.

L'effet détrimental ou bénéfique associé à l'énoncé de $\mathbf{a} \mathbf{b} \mathbf{c}$ est également lié au point de vue de l'observateur, à la manière dont il conçoit l'ordre des choses et ressent la variation relativement à ses attentes, soit qu'elle lui paraisse conforme à ses représentations (Ca tourne: «tout va bien, comme ça doit aller, tant mieux »), soit au contraire qu'il soit désagréablement surpris par la tournure que prennent les événements (Ob là là, ça tourne : «j'ai trop bu, je ne me sens pas bien » ou «il y a trop de tournants, c'est dangereux, j'ai peur»), ou qu'il attende que la variation débouche sur un résultat qui - par définition - n'est pas atteint (D'accord, ça tourne : « et alors? on n'avance pas!»).

La forme schématique proposée n'est qu'une esquisse: elle représente le résultat d'une investigation partielle énoncé dans des termes informels, en ceci qu'ils ne s'inscrivent pas rigoureusement dans le système conceptuel auquel sa tentative de construction est censée la rattacher. Oserai-je dire qu'elle tourne ? Oui, dans la mesure où, si elle paraît appropriée, elle n'échappe pas à la circularité : elle retrace ce que j’ai tiré des exemples considérés au départ et sa validité n’a été testée que par un retour simple à ces mêmes données; il eût fallu de surcroît en étudier soigneusement le déploiement dans les divers emplois auxquels elle prête ${ }^{18}$.

Il reste en outre à la confronter aux autres constructions, délibérément mises de côté dès le départ, du verbe tourner; elle devrait ensuite être vérifiée sur des paradigmes distributionnels étendus (quels $\mathrm{N}_{0}$ dans $N_{0}$ tourne / $N_{0}$ tourne sur soi-même, et quels $\mathrm{N}_{1}$ et prépositions ou adverbes dans les énoncés impliquant un complément ou un ajout), et sur des corpus systématiquement attestés. Le classement proposé en 2. s'est d'autre part fondé sur un nombre restreint de propriétés : tourner peut être non seulement intransitif mais aussi transitif et pronominal, il est apparenté à tour, tournant et également à tournis, tournement, tourne, et à des verbes tels que détourner, contourner, retourner, etc.

En somme - et heureusement - il reste beaucoup à faire ${ }^{19}$ !

\footnotetext{
${ }^{18}$ De plus, l'un des relecteurs anonymes m'objecte aimablement que la définition proposée est trop puissante, du fait qu'on pourrait l'appliquer sans trop de difficulté à Pierre rougit d'émotion.

${ }^{19}$ Je remercie pour leur infatigable disponibilité R. Camus, S. de Vogüé, J. Dubois, F. DuboisCharlier, J.-J. Franckel, F. Fresquet, D. Lebaud, K. Oguma, D. Paillard, P. Péroz, M. Sakhokia, F. Thuillier et les lecteurs anonymes de la revue qui m'ont conduite, par leurs remarques, à un peu plus de précision.
} 


\section{RÉFÉRENCES BIBLIOGRAPHIQUES}

ANSCOMBRE, J.-C., 1995, « La nature des topoï», in J.-C. Anscombre (dir.) Théorie des Topoï. Paris : Kimé, 49-84.

ANSCOMBRE, J.-C., 2002, «Mais/pourtant dans la contre-argumentation directe : raisonnement, généricité, lexique ». Linx, 46. Université de Paris X Nanterre, 115-132.

BONAMI, O., 1999, Les constructions du verbe : le cas des groupes prépositionnels argumentaux. Thèse de doctorat, Université de Paris 7.

BOONS, J.-P., GUILLET, A. \& LECLERE C., 1976, La structure des phrases simples en francais. Genève-Paris : Droz.

CADIOT, P. et NEMO, F., 1997, "Pour une sémiogénèse du nom », Langue française, 113. Paris : Larousse, 24-34.

CULIOLI, A., 1974 in 1999a, "Comment tenter de construire un modèle logique adéquat à la description des langues naturelles », Pour une linguistique de l'énonciation, tome 2. Paris : Ophrys, 53-66.

CULIOLI, A., 1979 in 1999a, «Conditions d'utilisation des données issues de plusieurs langues naturelles », Pour une linguistique de l'énonciation, tome 2. Paris : Ophrys, 67-82.

CULIOLI, A., 1982a in 1999b, «A propos de quelque », Pour une linguistique de l'énonciation, tome 3. Paris : Ophrys, 49-58.

CULIOLI, A., 1982b in 1999a, «Rôle des représentations métalinguistiques en syntaxe », Pour une linguistique de l'énonciation, tome 2. Paris : Ophrys, 84-95.

DENDALE, P. \& DE MULDER, W., 1997, «Les traits des emplois de la préposition spatiale sur», Faits de langues, 9. Paris : Ophrys, 211-220.

DENDALE, P. \& DE MULDER, W., 1998, «Contre et sur: du spatial au métaphorique ou inversement? », Verbum, XX : 4. Presses universitaires de Nancy, 405-434.

DE VOGUE, S., 1991, «La transitivité comme question théorique : querelle entre la Théorie des Positions de J.-C. Milner et la Théorie des Opérations Prédicatives et Énonciatives d'A. Culioli », Linx, 24. Université de Paris X Nanterre, 37-66.

DE VOGUE, S. et PAILLARD, D., 1997, «Identité lexicale et hétérogénéité de la variation co-textuelle. Le cas de suivre», in C. Guimier (éd.) Co-texte et calcul du sens. Caen : Presses universitaires de Caen, 41-62.

DE VOGUE, S. ; FRANCKEL, J.-J. et PAILLARD, D., 1989, «Extension de la distinction discret, dense, compact au domaine verbal», in J. David et G. Kleiber (éds) Termes massifs et termes comptables, Recherches linguistiques XIII. Université de Metz, 239-247.

DUBOIS, J. et DUBOIS-CHARLIER, F., 1996, Les verbes français. Paris : Larousse.

DUBOIS, J. et DUBOIS-CHARLIER, F., 2003, Structures verbales en français. Aix-en-Provence.

FRANCKEL, J.-J., 1998, «Référence, référenciation et valeurs référentielles », Sémiotiques, 15. Paris : Didier et CNRS, 61-84. 
FRANCKEL, J.-J., 2002, "Introduction », Le lexique : identité et variation, Langue française, 133. Paris : Larousse, 3-15.

FRANCKEL, J.-J., 2003, «De l'interprétation à la glose: vers une méthodologie de la reformulation », Conférence prononcée à Besançon, à paraître.

FRANCKEL, J.-J. et PAILLARD, D., 1991, «Discret, dense, compact : vers une typologie opératoire », Travaux de Linguistique et de Philologie, XXIX. Université de Strasbourg, 103136.

FRANÇOIS, J., 1989, Changement, causation, action. Genève-Paris : Droz.

GROSS, G. [dir.] , 1993, Sur le passif, Langages, 109. Paris : Larousse.

GROSS, M., 1975, Méthodes en syntaxe. Paris : Hermann.

GUILLAUME, G., 1971, Principes de linguistique théorique. Paris : Klincksieck.

HARRIS, S. Z., 1988, Language and Information. New York : Columbia University Press.

KLEIBER, G., 1997, «Sens, référence et existence : que faire de l'extralinguistique ? », Langages 127. Paris : Larousse, 9-37.

LEBAS, F. et CADIOT, P., 2003, «Monter et la constitution extrinsèque du référent », Langages 150. Paris : Larousse, 9-30.

LEEMAN, D., 1999, «L'unité lexicale dans la perspective harrissienne », Linx, 40. Université de Paris X Nanterre, 117-136.

LEEMAN, D., 2000 in 2004, «La préposition contre et les compléments de verbe (emplois non spatiaux) ", Colloque La médiation, Rouen. Actes à paraître aux Presses Universitaires de Rouen.

LEEMAN, D., 2001 in 2003, « Nom et verbe au regard de la préposition contre », Colloque Nom et Verbe : catégorisation et référence, Reims. Actes aux Presses universitaires de Reims, 139-160.

LEEMAN, D., 2002, La phrase complexe ; les subordinations. Bruxelles : De Boeck, Dpt Duculot.

MELIS, L. et DESMET, P., 2000, «La phrase et son analyse », Modèles linguistiques, XXXI : 2 (vol. 42). Université de Toulon et du Var, 79-145.

PAILLARD, D., 1988, «Fonctionnement du présent simple », Cabiers Jussieu : Nouvelles recherches sur le langage (numéro spécial). Paris, Université de Paris 7, 48-62.

PAILLARD, D., 2000, «À propos des verbes polysémiques », Syntaxe \& Sémantique, 2. Caen : Presses universitaires de Caen, 99-120.

PAILlARD, D., 2000 in 2002, «Prépositions et rection verbale », Colloque Prep’ 2000. Tel-Aviv : actes dans Travaux de linguistique, 44. Bruxelles, Duculot, 51-67.

RASTIER, F., 1991 rééd. 2001, Sémantique et recherches cognitives. Paris : PUF.

ROMERO-LOPES, M. C., 2002, «Identité et variation du verbe jouer», Langue française, 133. Paris : Larousse, 63-73.

SAUSSURE, F. de, 1916, Cours de linguistique générale. Paris : Payot. 
Danielle Leeman

THUILLIER, F., 2003, Systématique des emplois du verbe paraitre en français contemporain. Thèse de doctorat, Université de Paris 7.

VANDELOISE, C., 1986, L'espace en français. Paris : Seuil.

VANDELOISE, C., 1987, «La préposition à et le principe d'anticipation », Langue française, 76. Paris : Larousse, $77-111$.

VANDELOISE, C., 1993, «La préposition à pâlit-elle derrière toucher»? Langages, 110. Paris : Larousse, 107-127. 